\title{
$N$-BODY OBSERVABLES IN THE CALKIN ALGEBRA
}

\author{
JAN DEREZIŃSKI
}

\begin{abstract}
The commutators of many operators which are used in the phase space analysis of the $N$-body scattering are compact. This fact makes it possible to give a description of certain classes of such operators in terms of commutative $C^{*}$-algebras inside the Calkin algebra.
\end{abstract}

\section{INTRODUCTION}

The quantum evolution of a system of $N$ particles is usually described by selfadjoint operators on $L^{2}\left(\mathbb{R}^{N n}\right)$ of the form

$$
H=-\sum_{i=1}^{N} \frac{1}{2} \Delta_{i}+\sum_{i, j=1}^{N} v_{i j}\left(x_{i}-x_{j}\right)
$$

[RS1, CFKS]. Such operators are called $N$-body Schrödinger operators. If we try to study the scattering theory for such operators it is very useful to look at them "microlocally" [SigSof, De1]. A typical tool used in the "phase space analysis" of $N$-body systems are operators of the form

$$
h(H) Q(x /|x|) g(D),
$$

where $h(H)$ is a cutoff in the energy, $Q(x /|x|)$ is a cutoff in the configuration space, and $g(D)$ is a cutoff in the momentum. The phase space analysis of $N$-body systems is in a sense more difficult and more interesting than the usual microlocal analysis [Hö] because the potentials do not decay at infinity in certain directions of the configuration space. In particular, the position and momentum cutoffs that appear in operators of the form (2) used in the study of $N$-body systems usually satisfy certain geometric requirements reflecting the geometry of $N$-body potentials.

It turns out that the commutator of two operators of the form (2) (with certain natural conditions on $h, Q, g$ ) is compact. Consequently, the images of such operators inside the Calkin algebra form a commutative $C^{*}$-algebra. (Recall that the Calkin algebra is the algebra of bounded operators on a Hilbert space factored by the compact operators.)

It is possible to give a fairly detailed description of this commutative algebra inside the Calkin algebra. Namely, it is isomorphic to the space of continuous functions on a certain locally compact set $E S$ vanishing at infinity. The set $E S$

Received by the editors April 27, 1990.

1991 Mathematics Subject Classification. Primary 81U10; Secondary 46L60. 
is a certain geometric object whose definition takes into account the geometry of the $N$-body phase space and the spectra of all the subsystems. (The letters $E S$ stand for the energy shell.)

Another class of $N$-body observables that we study in this paper are operators of the form (2) multiplied by an operator that belongs to the pure point spectrum of cluster Hamiltonians. We can also describe the images of such operators inside the Calkin algebra in terms of $C^{*}$-algebras.

Originally, the formalism of this paper was intended as a tool in our study of asymptotic observables [De2]. Theorem 2.1 was an important preliminary step in our original proof of the main result of [De2]. This proof was based on Sigal's and Soffer's propagation theorem [SigSof, De1]. It turned out, however, that by using more recent ideas of [Graf] one can simplify the proof of the main result of [De2] significantly and avoid the application of Theorem 2.1. Nevertheless, we think that the formalism of the Calkin algebra presented in this paper reveals a certain nontrivial natural structure of $N$-body systems which is interesting in itself.

The proof of Theorem 2.1 has two major ingredients. One of them is the compactness of certain observables related to $N$-body systems. Facts of this sort are well known and are usually associated with the so-called geometric method. The geometric method consists roughly in looking at the $N$-body Schrödinger operators locally in the configuration space. An early result where this kind of thinking proved useful was the Hunziker-Van Winter-Zhislin theorem $[\mathrm{H}, \mathrm{VW}$, $\mathrm{Zh}, \mathrm{RS} 2]$. More applications of the geometric method can be found in [Sig, E1, Sim, CFKS]. The usefulness of cutoffs both in the configuration space and in the momentum was exploited by V. Enss (eg. [E2, E3]) and also in [SigSof].

The second ingredient of the proof of Theorem 2.1 is the use of certain basic properties of $C^{*}$-algebras, especially of the theorem on the tensor product of $C^{*}$-algebras [Ta].

\section{NOTATION AND THE MAIN RESUlt}

$B(\mathscr{H})$ will denote the $C^{*}$-algebra of bounded operators on a Hilbert space $\mathscr{H}$ and $C B(\mathscr{H})$ will denote the $C^{*}$-algebra of compact operators on $\mathscr{H}$. $C B(\mathscr{H})$ is a closed ideal in $B(\mathscr{H})$. Thus we can define the factor algebra $B(\mathscr{H}) / C B(\mathscr{H})$, which is called the Calkin algebra [Di]. If $B \in B(\mathscr{H})$ then $[B]_{\text {Cal }}$ will denote the image of $B$ in $\mathrm{Cal}(\mathscr{H})$. We will often drop the subscript Cal if it will not lead to a misunderstanding.

If $C$ is a selfadjoint operator then $\sigma(C)$ will denote its spectrum.

From the mathematical point of view it is convenient to replace the class of $N$-body Schrödinger operators with a more general class of operators first used by $\mathrm{S}$. Agmon $[\mathrm{A}, \mathrm{ABG}]$. In order to introduce this class we need some definitions.

A partially ordered set $(\mathscr{A}, \subset)$ with an operation $\cup$ such that if $a_{1} \subset b$ and $a_{2} \subset b$ then $a_{1} \cup a_{2} \subset b$ is called a semilattice. Throughout this paper we fix a certain finite semilattice $(\mathscr{A}, \subset, \cup)$. We will assume that there exists an element $a_{\min } \in \mathscr{A}$ such that $a_{\min } \subset a$ for all $a \in \mathscr{A}$. The element $\bigcup_{a \in \mathscr{A}} a$ will be denoted $a_{\max }$. We can also define the operation $\bigcap$ inside $\mathscr{A}$. Namely, we set $a_{1} \cap a_{2}:=\bigcup_{b \subset a_{1}, b \subset a_{2}} b$. (There is at least one such $b$, namely $a_{\min }$.) In this way $\mathscr{A}$ becomes a lattice. 
Throughout the paper $X$ will denote a fixed Euclidean space isomorphic to $\mathbb{R}^{n}$. We also fix a certain family $\left\{X_{a}: a \in \mathscr{A}\right\}$ of subspaces of $X$ indexed with the elements of the semilattice $\mathscr{A}$. We will assume that $a_{1} \subset a_{2}$ whenever $X_{a_{1}} \supset X_{a_{2}}, a_{1} \cup a_{2}=b$ whenever $X_{a_{1}} \cap X_{a_{2}}=X_{b}, X_{a_{\min }}=X$, and $X_{a_{\max }}=$ $\{0\}$. (In other words, $\left\{X_{a}: a \in \mathscr{A}\right\}$ is a family of subspaces closed wrt the intersection, and containing $X$ and $\{0\}$.) Note that $X_{a \cap b} \supset X_{a}+X_{b}$ (but sometimes the inclusion may be strict).

The orthogonal complement of $X_{a}$ in $X$ will be denoted $X^{a}$. The dual spaces to $X, X_{a}$, and $X^{a}$ will be denoted by $K, K_{a}$, and $K^{a}$ resp. $\pi_{a}$ will stand for the projection of $X$ onto $X_{a}$ and of $K$ onto $K_{a}$. $\pi^{a}$ will stand for the projection of $X$ onto $X^{a}$ and of $K$ onto $K^{a}$. We will often write $x_{a}$ instead of $\pi_{a} x, x^{a}$ instead of $\pi^{a} x$, etc.

$S$ will denote the unit sphere in $X$. There will also be special symbols for the following sets:

$$
Z_{a}:=\left(X_{a} \backslash \bigcup_{b \not \subset a} X_{b}\right) \cap S, \quad Y_{a}:=\left(X \backslash \bigcup_{b \not \subset a} X_{b}\right) \cap S .
$$

Note that $Y_{a}=\bigcup_{b \subset a} Z_{b}$ and $Y_{a_{\max }}=S$.

$D, D_{a}$, and $D^{a}$ will denote the operators $\frac{1}{i} \nabla, \frac{1}{i} \nabla_{a}$, and $\frac{1}{i} \nabla^{a}$ on $L^{2}(X)$. Likewise, $\Delta, \Delta_{a}$, and $\Delta^{a}$ denote the Laplacians corresponding to the variables $x, x_{a}$, and $x^{a}$.

The Euclidean norm of a vector $x \in X$ will be denoted $|x|$.

We will often abuse notation by identifying functions on the sphere with functions on $X$ homogeneous of degree zero.

We assume that for any $a \in \mathscr{A}$ we are given a real function $v_{a}$ on $X^{a}$ (which we will call the potential corresponding to the subsystem $a \in \mathscr{A}$ ). We will assume that $v_{a}\left(x^{a}\right)\left(1-\Delta^{a}\right)^{-1}$ is compact on $L^{2}\left(X^{a}\right)$.

We set

$$
V(x):=\sum_{a \in \mathscr{A}} v_{a}\left(\pi^{a} x\right) .
$$

Note that $V$ is bounded relatively to $-\Delta$ with the infinitesimal bound. We define $H$ to be the selfadjoint operator on $L^{2}(X)$ such that $\mathscr{D}(H)=\mathscr{D}(-\Delta)$ and $H=-\frac{1}{2} \Delta+V$.

If $a \in \mathscr{A}^{2}$ then we set

$$
V_{a}(x):=\sum_{b \subset a} v_{b}\left(\pi^{b} x\right) .
$$

We define $H_{a}$ to be the selfadjoint operator on $L^{2}(X)$ such that $\mathscr{D}\left(H_{a}\right)=$ $\mathscr{D}(-\Delta)$ and $H_{a}=-\frac{1}{2} \Delta+V_{a}$.

We may identify $L^{2}(X)$ with $L^{2}\left(X_{a}\right) \otimes L\left(X^{a}\right)$. Then we can write

$$
H_{a}=-\frac{1}{2} \Delta_{a} \otimes 1+1 \otimes H^{a},
$$

where $H^{a}$ is a certain selfadjoint operator on $L^{2}\left(X^{a}\right)$ that describes the inner degrees of freedom of the subsystem $a \in \mathscr{A}$. Clearly, $H^{a_{\max }}=H$.

$\mathscr{B}_{a}$ will denote the pure point spectrum of $H^{a} . \mathscr{B}_{a}^{\text {disc }}$ will denote the discrete spectrum of $H^{a}$. Note that $H^{a_{\min }}$ is defined on the one-dimensional space $L^{2}\left(X^{a_{\min }}\right)$ and equals zero. Therefore $\mathscr{B}_{a}=\{0\}$. 
$\mathscr{H}_{a \tau}$ will denote the subspace of eigenvectors of $H^{a}$ with eigenvalue $\tau . P_{a \tau}$ will denote the projections onto $\mathscr{H}_{a \tau}$.

$m$ will denote a certain fixed real number such that $-m \notin \sigma(H)$.

We will need a number of elementary concepts taken from the theory of commutative $C^{*}$-algebras [Di, Ta]. If $\Lambda$ is a topological space then $C(\Lambda)$ and $C_{0}(\Lambda)$ denote the $*$-algebras of continuous functions on $\Lambda$ and continuous functions on $\Lambda$ of compact support resp. If $\Lambda$ is a locally compact space then $C_{\infty}(\Lambda)$ denotes the $C^{*}$-algebra of continuous functions on $\Lambda$ that vanish at infinity. Note that if $\Lambda$ is compact then $C_{\infty}(\Lambda)=C(\Lambda)$.

It is well known that any commutative $C^{*}$-algebra with identity is isomorphic to $C(\Lambda)$ for some compact $\Lambda$. Any commutative $C^{*}$-algebra without identity is isomorphic to $C_{\infty}(\Lambda)$ for some locally compact but noncompact $\Lambda$.

If $\Lambda$ is locally compact but noncompact then $\Lambda^{\text {comp }}$ will denote the one-point compactification of $\Lambda$, that is, $\Lambda \cup\{\infty\}$ where $\infty$ is the "point at infinity." Note that if we adjoin the identity to $C_{\infty}(\Lambda)$ then we obtain an algebra canonically isomorphic to $C\left(\Lambda^{\text {comp }}\right)$. If $\Lambda$ is compact then we set $\Lambda^{\text {comp }}:=\Lambda$.

If $\Lambda_{1}$ and $\Lambda_{2}$ are compact spaces then any continuous map $\sigma: \Lambda_{1} \rightarrow \Lambda_{2}$ induces a $*$-homomorphism $\sigma^{*}: C\left(\Lambda_{2}\right) \rightarrow C\left(\Lambda_{1}\right)$ such that if $\psi \in C\left(\Lambda_{2}\right)$ then $\sigma^{*}(\psi)(\lambda):=\psi(\sigma(\lambda))$. More generally, if $\Lambda_{1}$ and $\Lambda_{2}$ are locally compact spaces then any continuous map $\sigma: \Lambda_{1}^{\text {comp }} \rightarrow \Lambda_{2}^{\text {comp }}$ such that $\sigma(\infty)=\infty$ induces a *-homomorphism $\sigma^{*}: C_{\infty}\left(\Lambda_{2}\right) \rightarrow C_{\infty}\left(\Lambda_{1}\right)$ in the same fashion.

If $\Xi$ is a closed subset of a locally compact space $\Lambda$ then $\Lambda \backslash \Xi$ is also locally compact. The $C^{*}$-algebra $C(\Lambda \backslash \Xi)$ can be identified with the subalgebra of $C_{\infty}(\Lambda)$ consisting of those $\psi \in C_{\infty}(\Lambda)$ that vanish at $\Xi$. Any closed ideal inside $C_{\infty}(\Lambda)$ is of the form of $C_{\infty}(\Lambda \backslash \Xi)$ for some closed $\Xi$.

For any $a \neq a_{\max }$ we define

$$
e s_{a}:=\left\{\left(k_{a}, \lambda\right) \in K_{a} \times \mathbb{R}: \lambda-\frac{1}{2} k_{a}^{2} \in \sigma\left(H^{a}\right)\right\}
$$

and

$$
E S:=\bigcup_{a \neq a_{\max }} Z_{a} \times e s_{a} .
$$

If $a \neq a_{\max }$ and $\tau \in \mathscr{B}_{a}$ then we define

$$
E S_{a \tau}:=Z_{a} \times\left\{\left(k_{a}, \lambda\right) \in K_{a} \times \mathbb{R}: \lambda-\frac{1}{2} k_{a}^{2}=\tau\right\} .
$$

We also define

$$
E S^{\mathrm{disc}}:=\bigcup_{a \neq a_{\max }} \bigcup_{\tau \in \mathscr{B}_{a}^{\mathrm{disc}}} E S_{a \tau} .
$$

Next we define the map

$$
k_{a}: E S \rightarrow\left(Y_{a} \times K_{a} \times \mathbb{R}\right)^{\mathrm{comp}}
$$

as follows. If $\left(x_{b}, k_{b}, \lambda\right) \in Z_{b} \times e s_{b}$ then

$$
k_{a}\left(x_{b}, k_{b}, \lambda\right):= \begin{cases}\left(x_{b}, \pi_{a} k_{b}, \lambda\right) & \text { if } b \subset a, \\ \infty & \text { otherwise. }\end{cases}
$$

We set $k_{a \tau}$ to be the restriction of $\ell_{a}$ to $E S_{a \tau}$ (or equivalently, the imbedding of $E S_{a \tau}$ in $\left.\left(Y_{a} \times K_{a} \times \mathbb{R}\right)^{\text {comp }}\right)$.

We endow $E S$ with the weakest topology such that the maps $k_{a}$ are continuous. Note that $E S$ becomes in this way a locally compact space. Let us 
also remark that if $\left(x_{a}, k_{a}, \lambda\right) \in Z_{a} \times e s_{a}$ then an example of a basis of neighborhoods of $\left(x_{a}, k_{a}, \lambda\right)$ in $E S$ can be given by $\left(k_{a}\right)^{-1}\left(\mathscr{U}_{1}, \mathscr{U}_{2}, \mathscr{U}_{3}\right)$, where $\mathscr{U}_{1}, \mathscr{U}_{2}, \mathscr{U}_{3}$, are neighborhoods of $x_{a}, k_{a}, \lambda$ in $Y_{a}, K_{a}, \mathbb{R}$ resp.

The following theorem is the main result of this paper.

Theorem 2.1. (a) Let $a \neq a_{\max }$. Then there exists a unique *-homomorphism $\tilde{\rho}_{a}$ from $C_{\infty}\left(Y_{a}, K_{a}, \mathbb{R}\right)$ to $\mathrm{Cal}\left(L^{2}(X)\right)$ that satisfies the following condition. Let $Q \in C_{\infty}\left(Y_{a}\right), g \in C_{\infty}\left(K_{a}\right)$, and $h \in C_{\infty}(\mathbb{R})$. Then

$$
\tilde{\rho}_{a}(Q \otimes g \otimes h)=\left[Q(x) g\left(D_{a}\right) h(H)\right]_{\mathrm{Cal}}=\left[Q(x) g\left(D_{a}\right) h\left(H_{a}\right)\right]_{\mathrm{Cal}} .
$$

(b) There exists a unique isometric *-homomorphism $\rho$ from $C_{\infty}(E S)$ to $\mathrm{Cal}\left(L^{2}(X)\right)$ such that if $\psi \in C_{\infty}\left(Y_{a} \times K_{a} \times \mathbb{R}\right)$ then $\rho\left(\mathscr{k}_{a}^{*}(\psi)\right)=\tilde{\rho}_{a}(\psi)$.

(c) Now assume additionally that $\tau \in \mathscr{B}_{a}$. Then there exists a unique *homomorphism $\tilde{\rho}_{a \tau}$ from $C B\left(\mathscr{H}_{a \tau}\right) \otimes C_{\infty}\left(Y_{a}, K_{a}, \mathbb{R}\right)$ to $\mathrm{Cal}\left(L^{2}(X)\right)$ that satisfies the following condition. Let $B \in C B\left(\mathscr{H}_{a \tau}\right), Q \in C_{\infty}\left(Y_{a}\right), g \in C_{\infty}\left(K_{a}\right)$, and $h \in C_{\infty}(\mathbb{R})$. Then

$$
\begin{aligned}
\tilde{\rho}_{a \tau}(B \otimes Q \otimes g \otimes h) & =\left[B Q(x) g\left(D_{a}\right) h(H)\right]_{\mathrm{Cal}} \\
& =\left[B Q(x) g\left(D_{a}\right) h\left(H_{a}\right)\right]_{\mathrm{Cal}} \\
& =\left[B Q\left(\pi_{a} x\right) g\left(D_{a}\right) h\left(H_{a}\right)\right]_{\mathrm{Cal}} .
\end{aligned}
$$

(d) There exists a unique isometric *-homomorphism $\rho_{a \tau}$ from $C B\left(\mathscr{H}_{a \tau}\right) \otimes$ $C_{\infty}\left(E S_{a \tau}\right)$ to $\mathrm{Cal}\left(L^{2}(X)\right)$ such that if $B \in C B\left(\mathscr{H}_{a \tau}\right)$ and $\psi \in C_{\infty}\left(Y_{a} \times K_{a} \times \mathbb{R}\right)$ then $\rho_{a \tau}\left(B \otimes \mathcal{K}_{a \tau}^{*}(\psi)\right)=\tilde{\rho}_{a \tau}(B \otimes \psi)$. 0 .

(e) If $C_{i} \in \operatorname{Ran} \rho_{a_{i} \tau_{i}}$, where $i=1,2$ and $\left(a_{1}, \tau_{1}\right) \notin\left(a_{2}, \tau_{2}\right)$, then $C_{1} C_{2}=$

(f) Let $\psi \in C_{\infty}(E S), B \in C B\left(\mathscr{H}_{a \tau}\right)$, and $\omega \in C_{\infty}\left(E S_{a \tau}\right)$. Then

$$
\rho(\psi) \rho_{a \tau}(B \otimes \omega)=\rho_{a \tau}(B \otimes \omega) \rho(\psi)=\rho_{a \tau}\left(B \otimes \omega \psi_{\mid E S_{a \tau}}\right) .
$$

(g) Let $\psi \in C_{\infty}\left(E S^{\text {disc }}\right)$. Then

$$
\rho(\psi)=\bigcup_{a \neq a_{\max }} \bigcup_{\tau \in \mathscr{B}_{a}^{\text {disc }}} \rho_{a \tau}\left(P_{a \tau} \otimes \psi_{\mid E S_{a \tau}}\right) .
$$

We can summarize (a), (b), (c), and (d) by drawing the following commutative diagrams:

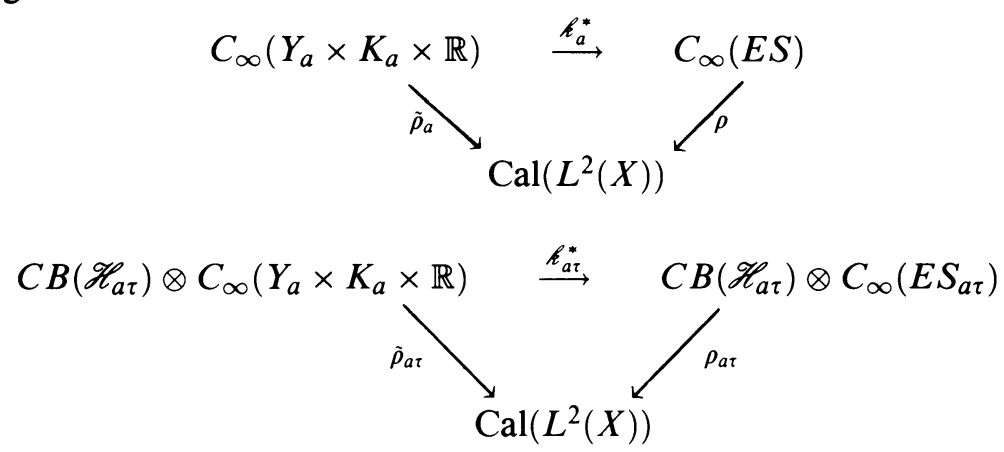

\section{Commutative $C^{*}$-algebras}

This section contains certain facts about commutative $C^{*}$-algebras that we will use in our paper. We start with a number of well-known results which we state without a proof [ $\mathrm{Di}, \mathrm{Ta}$ ]. In the sequel we will assume that $\Lambda$ is a locally compact set and $\Xi$ is its closed subset. 
Proposition 3.1. Let $\tilde{\rho}$ be a $*$-homomorphism of $C_{\infty}(\Lambda)$ into a $C^{*}$-algebra $\mathscr{C}$. Suppose that $C_{\infty}(\Lambda \backslash \Xi) \subset \operatorname{Ker} \tilde{\rho}$. Then there exists a unique *-homomorphism $\rho: C_{\infty}(\Xi) \rightarrow \mathscr{C}$ such that the following diagram commutes:

$$
\begin{gathered}
C_{\infty}(\Lambda) \rightarrow C_{\infty}(\boldsymbol{\Xi}) \\
\tilde{\rho} \searrow C^{\swarrow \rho}
\end{gathered}
$$

Proposition 3.2. If $\mathscr{C}$ is a closed ideal in $C_{\infty}(\Lambda)$ and for every $\lambda \in \Lambda \backslash \Xi$ there exists $\psi \in \mathscr{C}$ such that $\psi(\lambda) \neq 0$ then $C_{\infty}(\Lambda \backslash \Xi) \subset \mathscr{C}$.

Proposition 3.3. Iffor $i=1, \ldots, n$ we are given $*$-homomorphisms $\rho_{i}: C_{\infty}\left(\Lambda_{i}\right)$ $\rightarrow \mathscr{C}$ with commuting images then the formula

$$
\rho_{1} \otimes \cdots \otimes \rho_{n}\left(\psi_{1} \otimes \cdots \otimes \psi_{n}\right):=\rho_{1}\left(\psi_{1}\right) \cdots \rho_{n}\left(\psi_{n}\right)
$$

defines a *homomorphism $\rho_{1} \otimes \cdots \otimes \rho_{n}: C_{\infty}\left(\Lambda_{1} \times \cdots \times \Lambda_{n}\right) \rightarrow \mathscr{C}$.

The remaining part of this section will be devoted to a certain construction which will be used in the proof of our main result. Here we will describe this construction in general terms. The symbols $\mathscr{A}, S, Z_{a}$, and $Y_{a}$ that will be used in this section need not have the meaning that we ascribed to them in the previous section.

Let $\mathscr{A}$ be a finite lattice and $S$ be a compact set. We will assume that $S=\bigcup_{a \in \mathscr{A}} Z_{a}$, where the $Z_{a}$ are certain fixed disjoint subsets of $S$. We set $Y_{a}:=\bigcup_{b \subset a} Z_{b}$ and assume that the $Y_{a}$ are open in $S$ and noncompact if $a \neq a_{\max }$. For any $a \in \mathscr{A}$ we assume that $\Omega_{a}$ is a compact set and for any $b \subset a$ we are given a continuous map $\sigma_{a b}: \Omega_{b} \rightarrow \Omega_{a}$ such that if $a \subset b \subset c$ then $\sigma_{a b} \sigma_{b c}=\sigma_{a c}$. We set $\Lambda:=\bigcup_{a \in \mathscr{A}} Z_{a} \times \Omega_{a}$ and $\Lambda_{a}:=\left(Y_{a} \times \Omega_{a}\right)^{\text {comp }}$. We define a family of continuous maps $k_{a}: \Lambda \rightarrow \Lambda_{a}$ such that if $(x, \omega) \in Z_{b} \times \Omega_{b}$ then

$$
\mathscr{k}_{a}(x, \omega):= \begin{cases}\left(x, \sigma_{a b}(\omega)\right) & \text { if } b \subset a, \\ \infty & \text { otherwise }\end{cases}
$$

We equip $\Lambda$ with the weakest topology such that the $k_{a}$ are continuous.

Theorem 3.4. Suppose that $\mathscr{C}$ is a $C^{*}$-algebra, and both $\alpha: C(S) \rightarrow \mathscr{C}$ and $\zeta_{a}$ : $C\left(\Omega_{a}\right) \rightarrow \mathscr{C}$ are unital *-homomorphisms that satisfy the following conditions:

(1) If $Q \in C_{\infty}\left(Y_{a}\right)$ and $\psi \in C\left(\Omega_{a}\right)$ then $\alpha(\Omega) \zeta_{a}(\psi)=\zeta_{a}(\psi) \alpha(\Omega)$.

(2) If $b \subset a, Q \in C_{\infty}\left(Y_{b}\right)$, and $\psi \in C\left(\Omega_{a}\right)$ then

$$
\alpha(Q) \zeta_{a}(\psi)=\alpha(Q) \zeta_{b}\left(\sigma_{a b}^{*}(\psi)\right) .
$$

Then there exists a unique *-homomorphism $\rho: C(\Lambda) \rightarrow \mathscr{C}$ such that the following diagram commutes:

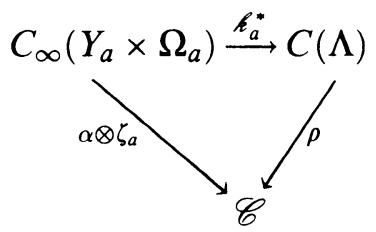

Proof. By (1) there exists a unique *-homomorphism $\alpha \otimes \zeta_{a}: C_{\infty}\left(Y_{a} \times \Omega_{a}\right) \rightarrow \mathscr{C}$ such that $\alpha \otimes \zeta_{a}(Q \otimes \psi)=\alpha(Q) \zeta_{a}(\psi)$. We extend $\alpha \otimes \zeta_{a}$ to a $*$-homomorphism $\rho_{a}: C_{\infty}\left(\Lambda_{a}\right) \rightarrow \mathscr{C}$ by setting $\rho_{a}(1):=1$. 
Now let $a_{1}, a_{2} \in \mathscr{A}, b=a_{1} \cap a_{2}, Q_{i} \in C_{\infty}\left(Y_{a_{i}}\right)$, and $\psi_{i} \in C\left(\Omega_{a_{i}}\right)$. Then

$$
\begin{aligned}
\rho_{a_{1}}\left(Q_{1} \otimes \psi_{1}\right) \rho_{a_{2}}\left(Q_{2} \otimes \psi_{2}\right) & =\zeta_{a_{1}}\left(\psi_{1}\right) \alpha\left(Q_{1}\right) \alpha\left(Q_{2}\right) \zeta_{a_{2}}\left(\psi_{2}\right) \\
& =\zeta_{a_{1}}\left(\psi_{1}\right) \alpha\left(Q_{1} Q_{2}\right) \zeta_{a_{2}}\left(\psi_{2}\right) .
\end{aligned}
$$

Note that $Y_{a_{1}} \cap Y_{a_{2}}=Y_{b}$. Therefore $Q_{1} Q_{2} \in C_{\infty}\left(Y_{a_{1}}\right) \cap C_{\infty}\left(Y_{a_{2}}\right)=C_{\infty}\left(Y_{b}\right)$. Thus (1) equals

$$
\zeta_{b}\left(\sigma_{a_{1} b}^{*}\left(\psi_{1}\right)\right) \alpha\left(Q_{1} Q_{2}\right) \zeta_{b}\left(\sigma_{a_{2} b}^{*}\left(\psi_{2}\right)\right)=\alpha\left(Q_{1} Q_{2}\right) \zeta_{b}\left(\sigma_{a_{1}}^{*}\left(\psi_{1}\right)\left(\sigma_{a_{2} b}^{*}\left(\psi_{2}\right)\right)\right.
$$

Clearly, $\rho_{a_{2}}\left(Q_{2} \otimes \psi_{2}\right) \rho_{a_{1}}\left(Q_{1} \otimes \psi_{1}\right)$ equals (2) as well. Therefore the images of $\rho_{a_{1}}$ and $\rho_{a_{2}}$ commute. Hence there exists a unique $*$-homomorphism $\bigotimes_{a \in \mathscr{A}} \rho_{a}$ : $C\left(\mathrm{X}_{a \in \mathscr{A}} \Lambda_{a}\right) \rightarrow \mathscr{C}$. We denote it by $\tilde{\rho}$. Clearly, Ker $\tilde{\rho}=C_{\infty}\left(\mathrm{X}_{a \in \mathscr{A}} \Lambda_{a} \backslash \Xi\right)$ for some closed $\Xi \subset \times_{a \in \mathscr{A}} \Lambda_{a}$. Let us fix our attention at a certain $\lambda=$ $\mathrm{X}_{a \in \mathscr{A}} \lambda_{a} \in \mathrm{X}_{a \in \mathscr{A}} \Lambda_{a}$. The remaining part of our proof will be broken into a number of steps.

Step 1. If $\lambda_{a_{\max }}=\left(y_{1}, \omega_{1}\right), \lambda_{b}=\left(y_{2}, \omega_{2}\right)$, and $y_{1} \neq y_{2}$ then $\lambda \notin \Xi$.

To see this choose open neighborhoods $\mathscr{U}_{1}$ and $\mathscr{U}_{2}$ of $y_{1}$ and $y_{2}$ in $Y_{a_{\max }}=$ $S$ and $Y_{b}$ resp. such that $\overline{\mathscr{U}_{1}} \cap \overline{\mathscr{U}_{2}}=\varnothing$. Let $Q_{1} \in C_{\infty}(S)$ and $Q_{2} \in C_{\infty}\left(Y_{b}\right)$ such that $\operatorname{supp} Q_{i} \subset \mathscr{U}_{i}$ and $Q_{i}\left(y_{i}\right) \neq 0$ for $i=1,2$. We can treat $Q_{1}$ as an element of $C\left(\Lambda_{a_{\max }}\right)$ and $Q_{2}$ as an element of $C\left(\Lambda_{b}\right)$. Now, $\tilde{\rho}\left(Q_{1} \otimes Q_{2}\right)=$ $\rho_{a_{\max }}\left(Q_{1}\right) \rho_{b}\left(Q_{2}\right)=\alpha\left(Q_{1}\right) \alpha\left(Q_{2}\right)=\alpha\left(Q_{1} Q_{2}\right)=0$.

Thus $Q_{1} \otimes Q_{2} \in \operatorname{Ker} \tilde{\rho}$ and $Q_{1} \otimes Q_{2}(\lambda) \notin 0$. By Proposition 3.2 this implies the statement of Step 1 .

Step 2. If $\lambda_{a_{\max }}=\left(y_{1}, \omega_{1}\right), y_{1} \in Z_{b}$, and $\lambda_{a}=\infty$ for some $a \supset b$, then $\lambda \notin \Xi$.

In fact, note that $y_{1} \in Y_{a}$. Thus we can find neighborhoods $\mathscr{U}_{1}$ and $\mathscr{U}_{2}$ of $y_{1}$ and $\infty$ in $S$ and $Y_{a}$ resp. such that $\overline{\mathscr{U}_{1}} \cap \overline{\mathscr{U}_{2}}=\varnothing$. Henceforth we argue as above.

So far we know that if $\lambda \in \Xi$ then there exists $b \in \mathscr{A}$ and $y \in Z_{b}$ such that

$$
\lambda_{a}= \begin{cases}\left(y, \omega_{a}\right) & \text { for } a \supset b, \\ \infty & \text { otherwise. }\end{cases}
$$

Step 3. If $\lambda$ is of the form (3) and $\omega_{a} \neq \sigma_{a b}\left(\omega_{b}\right)$ then $\lambda \notin \Xi$.

In fact, let $\mathscr{U}_{1}$ and $\mathscr{U}_{2}$ be neighborhoods of $\omega_{a}$ and $\sigma_{a b}\left(\omega_{b}\right)$ in $\Omega_{a}$ such that $\overline{\mathscr{U}_{1}} \cap \overline{\mathscr{U}_{2}}=\varnothing$. Let $\psi_{1}, \psi_{2} \in C\left(\Omega_{a}\right)$ such that $\psi_{1}\left(\omega_{a}\right) \neq 0, \psi_{2}\left(\sigma_{a b}\left(\omega_{b}\right)\right) \neq 0$, and supp $\psi_{i} \subset \mathscr{U}_{i}$. Let $Q \in C_{\infty}\left(Y_{b}\right)$ such that $Q(y) \neq 0$. We treat $Q \otimes \psi_{1}$ as an element of $C\left(\Lambda_{a}\right)$ and $Q \otimes \sigma_{a b}^{*}\left(\psi_{2}\right)$ as an element of $C\left(\Lambda_{b}\right)$. Now,

$$
\begin{aligned}
\tilde{\rho}(Q & \left.\otimes \psi_{1} \otimes Q \otimes \sigma_{a b}^{*}\left(\psi_{2}\right)\right) \\
& =\rho_{a}\left(Q \otimes \psi_{1}\right) \rho_{b}\left(Q \otimes \sigma_{a b}^{*}\left(\psi_{2}\right)\right)=\alpha(Q) \zeta_{a}\left(\psi_{1}\right) \zeta_{b}\left(\sigma_{a b}^{*}\left(\psi_{2}\right)\right) \alpha(Q) \\
& =\alpha(Q) \zeta_{a}\left(\psi_{1}\right) \zeta_{a}\left(\psi_{2}\right) \alpha(Q)=\alpha(Q) \zeta_{a}\left(\psi_{1} \psi_{2}\right) \alpha(Q)=0 .
\end{aligned}
$$

Thus $Q \otimes \psi_{1} \otimes Q \otimes \sigma_{a b}^{*}\left(\psi_{2}\right) \in \operatorname{Ker} \tilde{\rho}$ and $Q \otimes \psi_{1} \otimes Q \otimes \sigma_{a b}^{*}\left(\psi_{2}\right)(\lambda) \neq 0$. This ends the proof of Step 3 .

We conclude that if $\lambda \in \Xi$ then there exist $b \in \mathscr{A}, y \in Z_{b}$ and $\omega \in \Omega_{b}$ such that

$$
\lambda_{a}= \begin{cases}\left(y, \sigma_{a b}(\omega)\right) & \text { for } a \supset b, \\ \infty & \text { otherwise. }\end{cases}
$$


Now consider the map $k: \Lambda \rightarrow \times_{a \in \mathscr{A}} \Lambda_{a}$ such that $k(\lambda):=\times_{a \in \mathscr{A}} k_{a}(\lambda)$. Clearly, $k$ is continuous and injective. (4) means that $\Xi \subset \not(\Lambda)$.

To complete the proof we draw the following diagram:

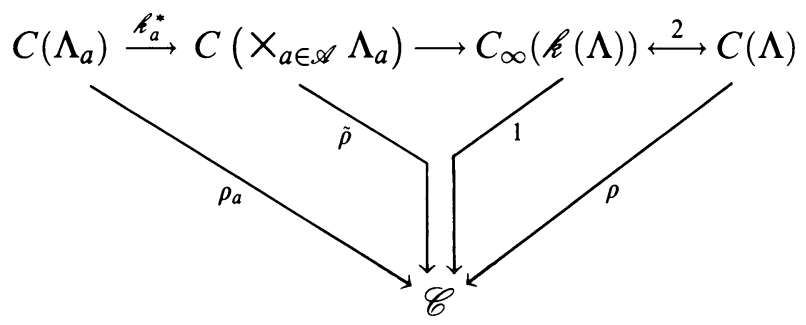

Arrow 1 exists by the previous reasoning and Proposition 3.1. Arrow 2 is an isomorphism. This proves the existence of $\rho$. Q.E.D.

\section{PROOF OF THE MAIN THEOREM}

Theorem 2.1 can be viewed as a fancy way of saying that certain operators are compact. Hence it should not be surprising that its proof is based on the following lemma, which belongs to the standard folklore of the geometric method.

Lemma 4.1. The following operators are compact.

(a) $\left[Q(x), g\left(D_{a}\right)\right] h(H)$ if $Q \in C(S), g \in C_{\infty}\left(K_{a}\right)$, and $h \in C_{\infty}(\mathbb{R})$.

(b) $Q(x)\left(h(H)-h\left(H_{a}\right)\right)$ if $Q \in C_{\infty}\left(Y_{a}\right)$ and $h \in C_{\infty}(\mathbb{R})$.

(c) $\left[Q(x), h\left(H_{a}\right)\right]$ if $Q \in C(S)$ and $h \in C_{\infty}(\mathbb{R})$.

Proof. (a) By the approximation argument we can assume that $Q \in C^{\infty}(S)$, $g \in C_{0}^{\infty}\left(K_{a}\right)$, and $h \in C_{0}(\mathbb{R})$. Then $\left[Q(x), g\left(D_{a}\right)\right]\langle x\rangle$ is bounded by a standard result from the calculus of pseudodifferential operators and $\langle x\rangle^{-1} h(H)$ is compact.

(b) We may assume that $Q \in C_{0}^{\infty}\left(Y_{a}\right)$ and $h \in C_{0}^{\infty}(\mathbb{R})$. Let $h_{1} \in C_{0}^{\infty}(\mathbb{R})$ such that $h_{1}\left((s+m)^{-1}\right)=h(s)$. We will use the following representation:

$$
h(H)=\frac{1}{2 \pi} \int d s \hat{h}_{1}(s) e^{i s(H+m)^{-1}} .
$$

The operator that we investigate is equal to

$$
\begin{array}{rl}
\frac{1}{2 \pi} \int d & s \hat{h}_{1}(s) s \int_{0}^{1} d \tau e^{i s \tau(H+m)^{-1}} \\
& \times Q(x)\left((H+m)^{-1}-\left(H_{a}+m\right)^{-1}\right) e^{i s(1-\tau)\left(H_{a}+m\right)^{-1}} \\
& +\frac{1}{2 \pi} \int d s \hat{h}_{1}(s) s^{2} \int_{0}^{1} d \tau \int_{0}^{1} d \tau_{1} e^{i s \tau(H+m)^{-1}}\left[(H+m)^{-1}, Q(x)\right] \\
& \times e^{i s\left(\tau_{1}-\tau\right)\left(H_{a}+m\right)^{-1}}\left((H+m)^{-1}-\left(H_{a}+m\right)^{-1}\right) e^{i s\left(1-\tau_{1}\right)\left(H_{a}+m\right)^{-1}}
\end{array}
$$

It is easy to show that $\left((H+m)^{-1}-\left(H_{a}+m\right)^{-1}\right)$ and $\left[(H+m)^{-1}, Q(x)\right]$ are compact. This implies the compactness of the above expression.

(c) We can assume that $Q \in C^{\infty}(S)$ and $h \in C_{0}^{\infty}(\mathbb{R})$. Then we argue similarly as above. Q.E.D.

Proof of Theorem 2.1(a) and (b). The proof is based on Theorem 3.4. We assume that the symbols $S, \mathscr{A}, Y_{a}$ and $Z_{a}$ have the meaning defined in $\S 2$. We 
set $\mathscr{C}:=\operatorname{Cal}\left(L^{2}\right)$ and $\Omega_{a}:=e s_{a}^{\text {comp }}$, and if $b \subset a$ then $\sigma_{a b}: e s_{b}^{\text {comp }} \rightarrow e s_{a}^{\text {comp }}$ is defined by $\sigma_{a b}\left(k_{b}, \lambda\right):=\left(\pi_{a} k_{b}, \lambda\right)$ and $\sigma_{a b}(\infty):=\infty$. (The map is well defined by the HZV theorem [RS2].) We also define

$$
C(S) \ni Q \rightarrow \alpha(Q):=[Q(x)]_{\mathrm{Cal}} \in \operatorname{Cal}\left(L^{2}(X)\right) .
$$

Since es $_{a}$ is the joint spectrum of the commuting operators $D_{a}$ and $H_{a}$, we can define

$$
C\left(e s_{a}^{\mathrm{comp}}\right) \ni \psi \rightarrow \zeta_{a}(\psi):=\left[\psi\left(D_{a}, H_{a}\right)\right]_{\mathrm{Cal}} \in \operatorname{Cal}\left(L^{2}(X)\right) .
$$

Note that if $g \in C_{\infty}\left(K_{a}\right)$ and $h \in C_{\infty}(\mathbb{R})$ then

$$
\zeta_{a}\left(g \otimes h_{\mid e s_{a}}\right)=\left[g\left(D_{a}\right) h\left(H_{a}\right)\right]_{\mathrm{Cal}} \text {. }
$$

We need to check the conditions of Theorem 3.4. It is enough to assume that $\psi$ is of the form $\psi=g \otimes h_{\mid e s_{a}}$, where $g \in C_{\infty}\left(K_{a}\right)$ and $h \in C_{\infty}(\mathbb{R})$. By Lemma 4.1(a) and (c) if $Q \in C(S)$ then $\left[Q(x), g\left(D_{a}\right) h\left(H_{a}\right)\right]$ is compact. Thus $\alpha(Q) \zeta_{a}(\psi)=\zeta_{a}(\psi) \alpha(Q)$.

Furthermore, if $b \subset a$ and $Q \in C_{\infty}\left(Y_{b}\right)$ then by Lemma 4.1(a), (b) the operator $Q(x) g\left(D_{a}\right) h\left(H_{a}\right)-Q(x) g\left(D_{a}\right) h\left(H_{b}\right)$ is compact. Hence $\alpha(Q) \zeta_{a}(\psi)=$ $\alpha(Q) \zeta_{b}\left(\sigma_{a b}^{*}(\psi)\right)$.

To complete the verification of the hypotheses of Theorem 3.5 it remains to check them for $\psi=1$, which is trivial.

Now let

$$
\Lambda:=\bigcup_{a \neq a_{\max }} Z_{a} \times e s_{a}^{\mathrm{comp}} .
$$

Clearly, $E S$ is a subset of $\Lambda$. Recall that we defined $k_{a}$ to be a map from $E S$ to $\left(Y_{a} \times K_{a} \times \mathbb{R}\right)^{\text {comp }}$. Let $k_{a}^{1}$ denote the map from $\Lambda$ to $\Lambda_{a}=\left(Y_{a} \times e s_{a}^{\text {comp }}\right)^{\text {comp }}$ which coincides with $k_{a}$ on $E S$ and is equal to $\infty$ on $\Lambda \backslash E S$.

Now Theorem 3.4 guarantees the existence of a $*$-homomorphism $\rho$ from $C(\Lambda)$ to $\mathrm{Cal}\left(L^{2}(X)\right)$ with the properties that can be summarized by the following diagram:

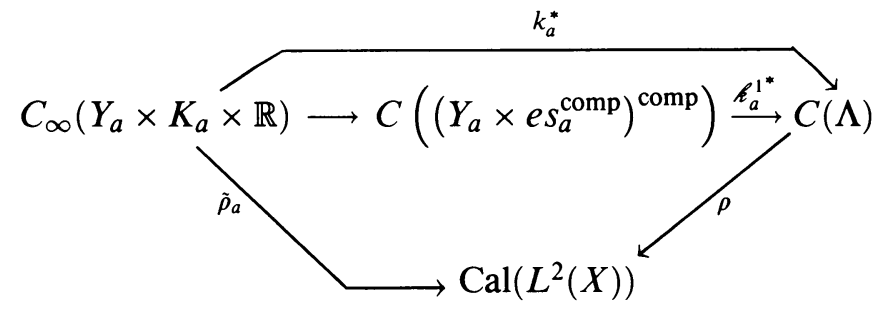

Next note that the images $k_{a}^{*}\left(C_{\infty}\left(Y_{a} \times K_{a} \times \mathbb{R}\right)\right)$ are contained in $C_{\infty}(E S)$ (which is a subalgebra of $C(\Lambda))$. Therefore it we restrict $\rho$ to $C_{\infty}(E S)$ then we can draw the diagram (2) of $\S 2$. Thus defined $\rho$ is unique because the images of $k_{a}^{*}$ generate $C_{\infty}(E S)$.

Now let us prove that $\rho$ is isometric. Let $\left(y_{a}, p_{a}, \mu\right) \in Z_{a} \times e s_{a}$. Fix $Q \in C_{\infty}\left(Y_{a}\right), g \in C\left(K_{a}\right)$, and $h \in C_{\infty}(\mathbb{R})$ such that $Q\left(y_{a}\right) g\left(p_{a}\right) h(\mu) \neq 0$. Clearly,

Hence

$$
\mathrm{s}_{t \rightarrow \infty} \lim ^{i t y_{a} D_{a}} Q(x) e^{-i t y_{a} D_{a}}=Q\left(y_{a}\right) .
$$

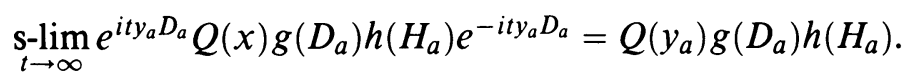


Therefore

$$
\left\|\left[Q(x) g\left(D_{a}\right) h\left(H_{a}\right)\right]_{\text {Cal }}\right\| \geq Q\left(y_{a}\right) g\left(p_{a}\right) h(\mu) .
$$

This means that $\left\|\rho\left(k_{a}^{*}(Q \otimes g \otimes h)\right)\right\|>0$. Consequently $\operatorname{Ker} \rho=\{0\}$. This implies the isometricity of $\rho$ and ends the proof of (a) and (b). Q.E.D.

To proceed further with the proof of Theorem 2.1 we will need the following lemma.

Lemma 4.2. Let $B \in C B\left(\mathscr{H}_{a \tau}\right), Q \in C(S)$, and $h \in C_{\infty}(\mathbb{R})$. Then

$$
\left[B Q(x) h\left(H_{a}\right)\right]=\left[B Q\left(\pi_{a} x\right) h\left(H_{a}\right)\right]=\left[h\left(H_{a}\right) B Q\left(\pi_{a} x\right)\right] .
$$

Proof. We set $\chi_{T}(s)=1$ if $|s|<T$, and $\chi_{T}(s)=0$ if $|s| \geq T$. If $\varepsilon>0$ then we will find $T>0$ such that $\left\|B\left(1-\chi_{T}\left(\left|x^{a}\right|\right)\right)\right\|<\varepsilon$. We will also find $T_{1}$ such that $\left\|\chi_{T}\left(\left|x^{a}\right|\right)\left(1-\chi_{T_{1}}\left(\left|x_{a}\right|\right)\right)\left(Q(x)-Q\left(x_{a}\right)\right)\right\|<\varepsilon$. Therefore up to an arbitrarily small error $B\left(Q(x)-Q\left(x_{a}\right)\right)$ is equal to $B\left(Q(x)-Q\left(x_{a}\right)\right) \chi_{T}\left(\left|x^{a}\right|\right) \chi_{T_{1}}\left(\left|x_{a}\right|\right)$. But $\chi_{T}\left(\left|x^{a}\right|\right) \chi_{T_{1}}\left(\left|x_{a}\right|\right) h\left(H_{a}\right)$ is compact. This proves the first equality.

To prove the second equality note that by Lemma 4.1(c) it is enough to show that

$$
h\left(H_{a}\right) B\left(Q(x)-Q\left(x_{a}\right)\right)
$$

is compact. As before we can replace (1) up to an arbitrarily small error with

$$
h\left(H_{a}\right) B\left(Q(x)-Q\left(x_{a}\right)\right) \chi_{T}\left(\left|x^{a}\right|\right) \chi_{T_{1}}\left(\left|x_{a}\right|\right) .
$$

Now

$$
h\left(H_{a}\right) B \chi_{T_{1}}\left(\left|x_{a}\right|\right)=h\left(H_{a}\right)\left(-\Delta_{a}+1\right)\left(-\Delta_{a}+1\right)^{-1} \chi_{T_{1}}\left(\left|x_{a}\right|\right) B
$$

is compact. Q.E.D.

Proof of Theorem 2.1(c) and (d). Consider the homomorphisms

$$
C B\left(\mathscr{H}_{a \tau}\right) \ni B \rightarrow[B] \in \operatorname{Cal}\left(L^{2}(X)\right)
$$

and

$$
C_{\infty}\left(Y_{a}, K_{a}, \mathbb{R}\right) \ni \psi \rightarrow \tilde{\rho}_{a}(\psi) \in \operatorname{Cal}\left(L^{2}(X)\right) .
$$

First note that their images commute. To see this it is enough to consider $\psi$ of the form $Q \otimes g \otimes h$, where $Q \in C_{\infty}\left(Y_{a}\right), g \in C\left(K_{a}\right)$, and $h \in C_{\infty}(\mathbb{R})$. Then by Lemma 4.2

$$
\begin{aligned}
{[B] \tilde{\rho}_{a}(\psi) } & =\left[B Q(x) g\left(D_{a}\right) h\left(H_{a}\right)\right]=\left[B Q\left(x_{a}\right) h\left(H_{a}\right) g\left(D_{a}\right)\right] \\
& =\left[Q\left(x_{a}\right) B h\left(H_{a}\right) g\left(D_{a}\right)\right]=\left[Q(x) B h\left(H_{a}\right) g\left(D_{a}\right)\right]=\tilde{\rho}_{a}(\psi)[B] .
\end{aligned}
$$

This proves the existence of $\tilde{\rho}_{a \tau}$.

Next note that if $g\left(k_{a}\right) h\left(\frac{1}{2} k_{a}^{2}+\tau\right)=0$ for $k_{a} \in K_{a}$ then $B g\left(D_{a}\right) h\left(H_{a}\right)=0$. Likewise, if $Q=0$ on $Z_{a}$ then by Lemma 4.2

$$
\left[B Q(x) h\left(H_{a}\right)\right]=\left[B Q\left(x_{a}\right) h\left(H_{a}\right)\right]=0 .
$$

Hence if $\psi=0$ on $E S_{a \tau}$ then $B \otimes \psi \in \operatorname{Ker} \tilde{\rho}_{a \tau}$. This implies the existence of $\rho_{a \tau}$.

Proof of Theorem 2.1(e). If $a_{1}=a_{2}$ and $\tau_{1} \neq \tau_{2}$ then the statement follows from the spectral theorem. Assume that $a_{1} \not \subset a_{2}$. It is enough to consider $B_{i} \in$ 
$C B\left(\mathscr{H}_{a_{i} \tau_{i}}\right)$ and $\omega_{i}=\ell_{a_{i} \tau_{i}}^{*}$ where $Q_{i} \in C_{\infty}\left(Y_{a_{i}}\right), g_{i} \in C\left(K_{a_{i}}\right)$, and $h_{i} \in C_{\infty}(\mathbb{R})$. Now

$$
\begin{aligned}
\rho_{a_{1} \tau_{1}} & \left(B_{1} \otimes \omega_{1}\right) \rho_{a_{2} \tau_{2}}\left(B_{2} \otimes \omega_{2}\right) \\
& =\left[B_{1} h_{1}(H) g\left(D_{a_{1}}\right) Q_{1}(x) Q_{2}(x) g\left(D_{a_{2}}\right) h_{2}(H) B_{2}\right] .
\end{aligned}
$$

Note that $Q_{2}=0$ on $Z_{a_{2}}$. For any $\varepsilon>0$ we can change $Q_{1}$ outside $Z_{a_{1}}$ such that $\left|Q_{1} Q_{2}\right| \leq \varepsilon$. Therefore the norm of (2) can be made arbitrarily small. Consequently, (2) is zero. Q.E.D.

Proof of (f). It is enough to assume that $\psi=k_{b}^{*}(Q \otimes g \otimes h)$, where $Q \in$ $C_{\infty}\left(Y_{b}\right), g \in C_{\infty}\left(K_{b}\right)$, and $h \in C_{\infty}(\mathbb{R})$. Likewise we can assume that $\omega=$ $k_{a \tau}^{*}\left(Q_{1} \otimes g_{1} \otimes h_{1}\right)$, where $Q_{1} \in C_{\infty}\left(Y_{a}\right), g_{1} \in C_{\infty}\left(K_{a}\right)$, and $h_{1} \in C_{\infty}(\mathbb{R})$. Now,

$$
\rho_{a \tau}(B \otimes \omega) \rho(\psi)=B Q_{1}(x) Q(x) g_{1}\left(D_{a}\right) g\left(D_{b}\right) h_{1}(H) h(H) \text {. }
$$

If $b \supset a$ then this is equal to

$$
\tilde{\rho}_{a \tau}\left(B \otimes Q_{1} Q \otimes g_{1} \pi_{a}^{*}(g) \otimes h_{1} h\right)=\rho_{a \tau}\left(B \otimes \omega \psi_{\mid E S_{a \tau}}\right) .
$$

If $b \not \supset a$ then (3) is zero because $Q=0$ on $Z_{a}$. Q.E.D.

Proof of $(\mathrm{g})$. By (f) the set of those $\psi \in C_{\infty}(E S)$ that satisfy (1) of $\S 2$ is an ideal in $C_{\infty}(E S)$. It is obviously closed. We will show that $C_{\infty}\left(E S^{\text {disc }}\right)$ is contained in this ideal.

Let $\left(y_{a}, p_{a}, \mu\right) \in Z_{a} \times e s_{a} \cap E S^{\text {disc }}$. Choose $Q \in C_{\infty}\left(Y_{a}\right), g \in C\left(K_{a}\right)$, and $h \in C_{\infty}(\mathbb{R})$ such that $Q\left(y_{a}\right) g\left(p_{a}\right) h(\mu) \neq 0$, and if $\left(k_{a}, \lambda\right) \in \operatorname{supp} g \times \operatorname{supp} h$ then $\lambda-\frac{1}{2} k_{a}^{2} \notin \sigma_{\mathrm{ess}}\left(H^{a}\right)$. Set $\psi=: k_{a}^{*}(Q \otimes g \otimes h)$. Then

$$
\begin{aligned}
\rho(\psi) & =\left[Q(x) g\left(D_{a}\right) h\left(H_{a}\right)\right] \\
& =\sum_{\tau \in \mathscr{B}_{a}^{\text {disc }}}\left[Q(x) g\left(D_{a}\right) h\left(\frac{1}{2} D_{a}^{2}+\tau\right) P_{a \tau}\right]=\sum_{\tau \in \mathscr{B}_{a}^{\text {disc }}} \rho_{a \tau}\left(P_{a \tau} \otimes \psi_{\mid E S_{a \tau}}\right) .
\end{aligned}
$$

Q.E.D.

\section{REFERENCES}

[A] S. Agmon, Lectures on the exponential decay of solutions of second order elliptic equations, Princeton Univ. Press, Princeton, N.J., 1982.

[ABG] W. O. Amrein, A. M. Boutet de Monvel-Berthier, and V. Georgescu, Notes on the N-body problem, preprint, Geneve, 1989.

[CFKS] H. L. Cycon, R. Froese, W. Kirsch, and B. Simon, Schrödinger operators with applications to quantum mechanics and global geometry, Springer, Berlin-Heidelberg-New York, 1987.

[De1] J. Dereziński, $A$ new proof of the propagation theorem for $N$-body quantum systems, Comm. Math. Phys. 122 (1989), 203-231.

[De2] _ Asymptotic observables in the long range $N$-body scattering theory, Proc. Summer School in Brasov, 1989 (to appear).

[Di] J. Dixmier, Les $C^{*}$-algebres et leurs representations, Gauthier-Villars, Paris, 1969.

[E1] V. Enss, A note on Hunziker's theorem, Comm. Math. Phys. 52 (1977), 233-238.

[E2] _ Quantum scattering theory of two-and three-body systems with potentials of short and long range, Schrödinger Operators (S. Graffi, ed.), Lecture Notes in Math., vol. 1159, Springer, Berlin-Heidelberg-New York, 1985.

[E3] _- Introduction to asymptotic observables for multiparticle quantum scattering, Proc. Sympos. Schrödinger Operators (Aarhus, 1985), (E. Balslev, ed.), Lecture Notes in Math., Springer, 1986. 
[Graf] G. M. Graf, Asymptotic completeness for $N$-body short-range systems: a new proof, preprint, 1989.

[Hu] W. Hunziker, On the spectra of Schrödinger multiparticle Hamiltonians, Helv. Phys. Acta 39 (1966), 451-462.

[RS1] M. Reed and B. Simon, Methods of modern mathematical physics. III: Scattering theory, Academic Press, London, 1979.

[RS2] _ Methods of modern mathematical physics. IV: Analysis of operators, Academic Press, London, 1978.

[Sig] I. M. Sigal, Geometric methods in the quantum many-body problem. Nonexistence of very negative ions, Comm. Math. Phys. 85 (1982), 309-324.

[SigSof] I. M. Sigal and A. Soffer, The N-particle scattering problem: asymptotic completeness for short range systems, Anal. Math. 125 (1987), 35-108.

[Sim] B. Simon, Geometric methods in multiparticle quantum systems, Comm. Math. Phys. 55 (1977), 259-274; Math. Phys. 58 (1978) 205-210.

[Ta] M. Takesaki, Theory of operator algebras. I, Springer, Berlin-Heidelberg-New York, 1979.

[VW] C. Van Winter, Theory of finite systems of particles. I, Mat. Fys. Skr. Danske Vid. Selsk. 1 (1964), 1-60.

[Zhi] G. M. Zhislin, Investigations of the spectrum of the Schrödinger operator for a many body system, Trudy Moskov. Mat. Obshch. 9 (1960), 81-128.

Department of Mathematical Methods in Physics, Warsaw University, Hoża 74, PL00-682 WARsZawa, Poland

E-mail address: derezins@plearn.bitnet 